\title{
Comprehensive in vitro characterization of the LSD1 small molecule inhibitor class in oncology
}

Natalia Sacilotto $\xi \perp$, Paola Dessanti $\xi \perp$, Michele M.P. Lufino $\xi$, Alberto Ortega $\xi$, Alejandra Rodríguez-Gimeno $\xi$,

Jordi Salas $\xi$, Tamara Maes $\xi$, Carlos Buesa $\xi$, Cristina Mascaró $\xi^{*}$, Robert Soliva $\xi^{*}$

$\xi$ Oryzon Genomics S.A. Carrer Sant Ferran 74, Cornellà de Llobregat, 08940, Barcelona, Spain.

$\perp$ These authors contributed equally.

* Corresponding authors

KEYWORDS. LSD1 inhibitors, cancer, Acute Myeloid Leukemia, Small cell lung cancer 
Table of Contents for Supplementary information

Figures:

Figure S1. Jump dilution analysis of reversible LSD1 inhibitors

Figure S2. Dose-response curves of MAO-A and MAO-B inhibition for selected tool compounds

Figure S3. Efficacy in AML

Tables:

Table S1. Stability of compounds in aqueous buffer at $37^{\circ} \mathrm{C}$

Table S2. Kinetic solubility, inhibition of LSD1 and other FAD dependent enzymes

Table S3. Kinetic parameters of LSD1 irreversible inhibition

Table S4. EC 50 viability values for AML and SCLC cell lines

Table S5. EC50 differentiation values for the AML cell line THP1 


\section{SUPPLEMENTARY FIGURES}

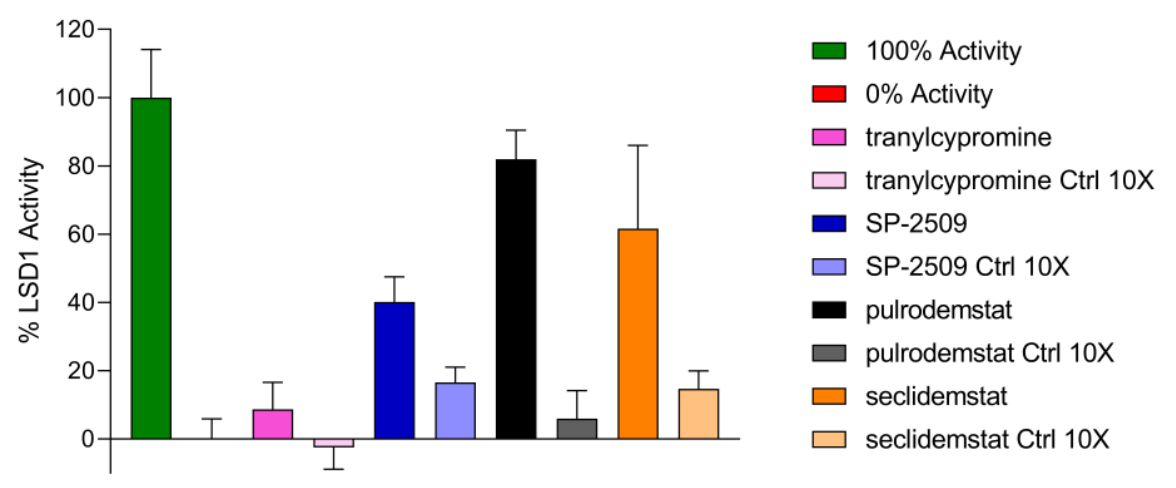

Figure S1. Jump dilution analysis of reversible LSD1 inhibitors. The recovery of LSD1 activity upon jump dilution is reported as a percentage of the activity of LSD1 incubated and diluted in the absence of inhibitor (100\% Activity). As a control, the percentage recovery of activity of LSD1 incubated with each compound and diluted with 10-fold the $\mathrm{IC}_{50}$ was determined (Ctrl 10X). TCP was used as a control of no recovery upon jump dilution. Data represented as mean \pm St. Dev. of $N=3, n=1$.

A

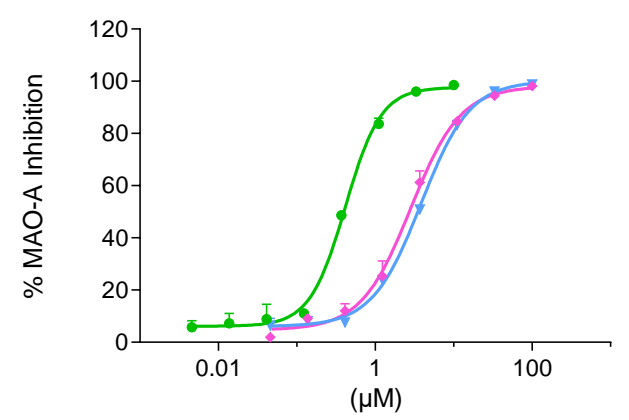

B

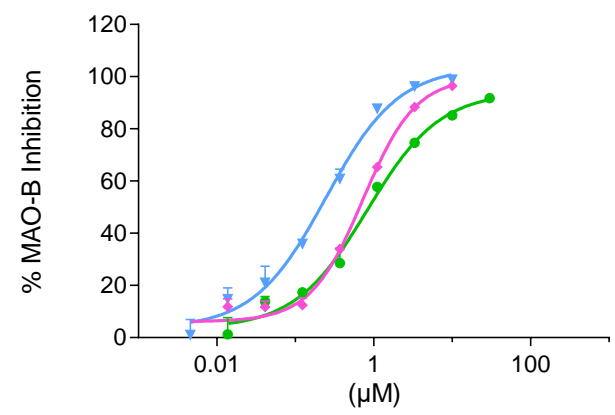

$\rightarrow$ tranylcypromine

$\rightarrow$ phenelzine

$\rightarrow$ pargyline

Figure S2. Dose-response curves of MAO-A (A) and MAO-B (B) inhibition for selected tool compounds. Data are represented as mean \pm St. Dev. of $N=2, n=2$. 
A

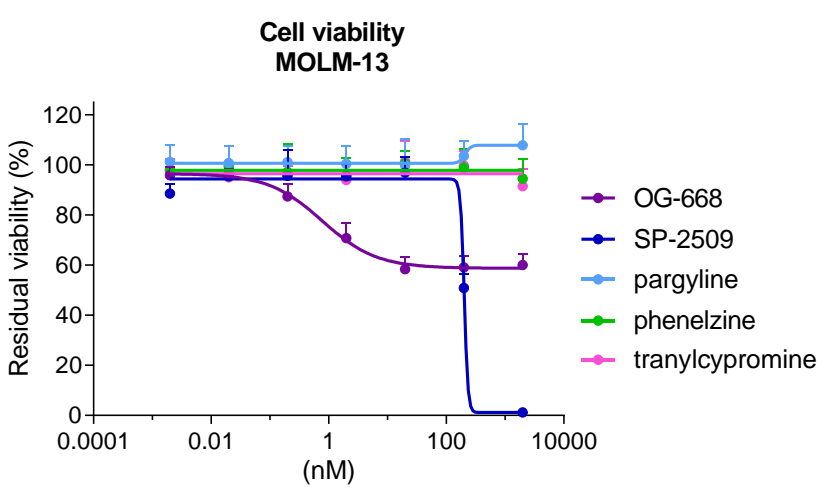

B

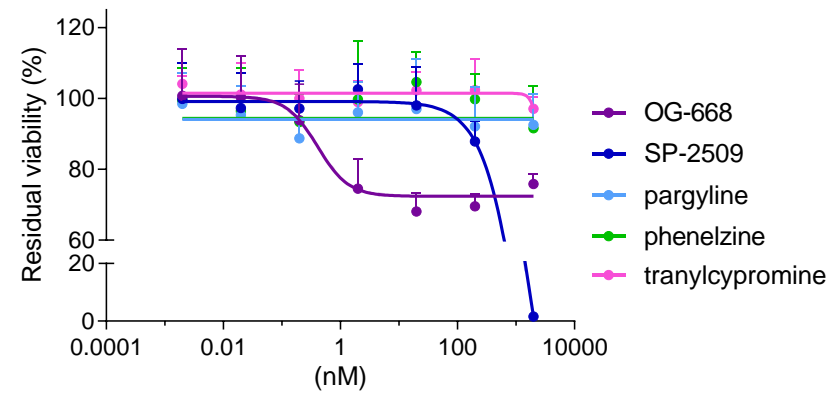

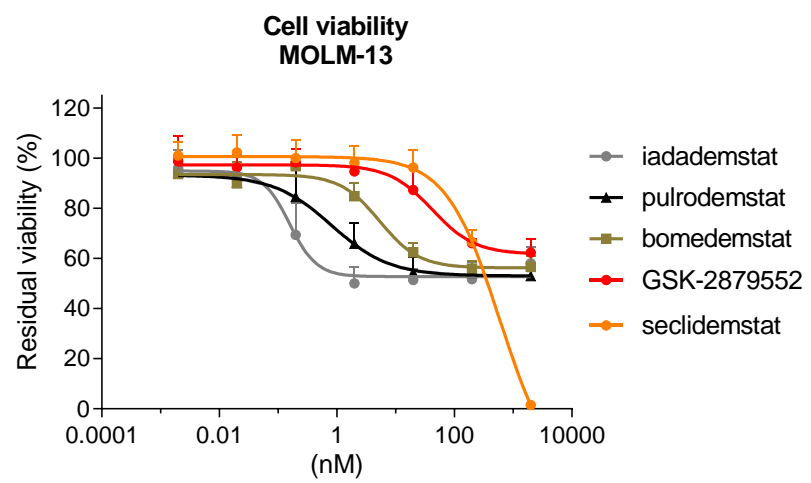

Cell viability

$\operatorname{MV}(4 ; 11)$

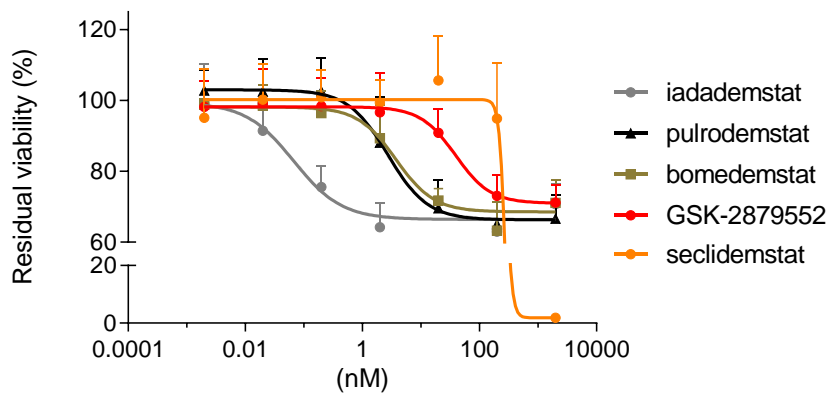

Figure S3. Efficacy in AML. Effect of tool (left panels) and clinical stage (right panels) LSD1 inhibitors on cell viability in the AML cell line MOLM13 (A) and MV(4;11) (B) following $96 \mathrm{hr}$ incubation at concentrations ranging from 0.002 to $2,000 \mathrm{nM}$. All data are expressed as relative to the vehicle condition and represented as mean \pm St. Dev of $\mathrm{N}=2, \mathrm{n}=3$. 


\begin{tabular}{cccc}
\hline Compound & $\begin{array}{c}\mathrm{T}=0 \text { days } \\
\text { purity }(\%)^{\mathrm{a}}\end{array}$ & $\begin{array}{c}\mathrm{T}=4 \text { days } \\
\text { purity }(\%)^{\mathrm{a}}\end{array}$ & $\begin{array}{c}\mathrm{T}=6 \text { days } \\
\text { purity }(\%)^{\mathrm{a}}\end{array}$ \\
\hline $\begin{array}{c}\text { phenelzine } \\
\text { tranylcypromine }\end{array}$ & 96 & 97 & 95 \\
pargyline & 94 & 5 & 4 \\
OG-668 & 97 & 98 & 95 \\
SP-2509 & 88 & 96 & 95 \\
pulrodemstat & 98 & 74 & 68 \\
GSK-2879552 & 99 & 98 & 92 \\
bomedemstat & 100 & 98 & 96 \\
seclidemstat & 88 & 95 & 93 \\
iadademstat & 99 & 74 & 91 \\
\hline
\end{tabular}

Table S1. Stability of compounds in aqueous buffer at $37^{\circ} \mathrm{C} .{ }^{\text {a }}$ Purity values obtained by LCMS. 


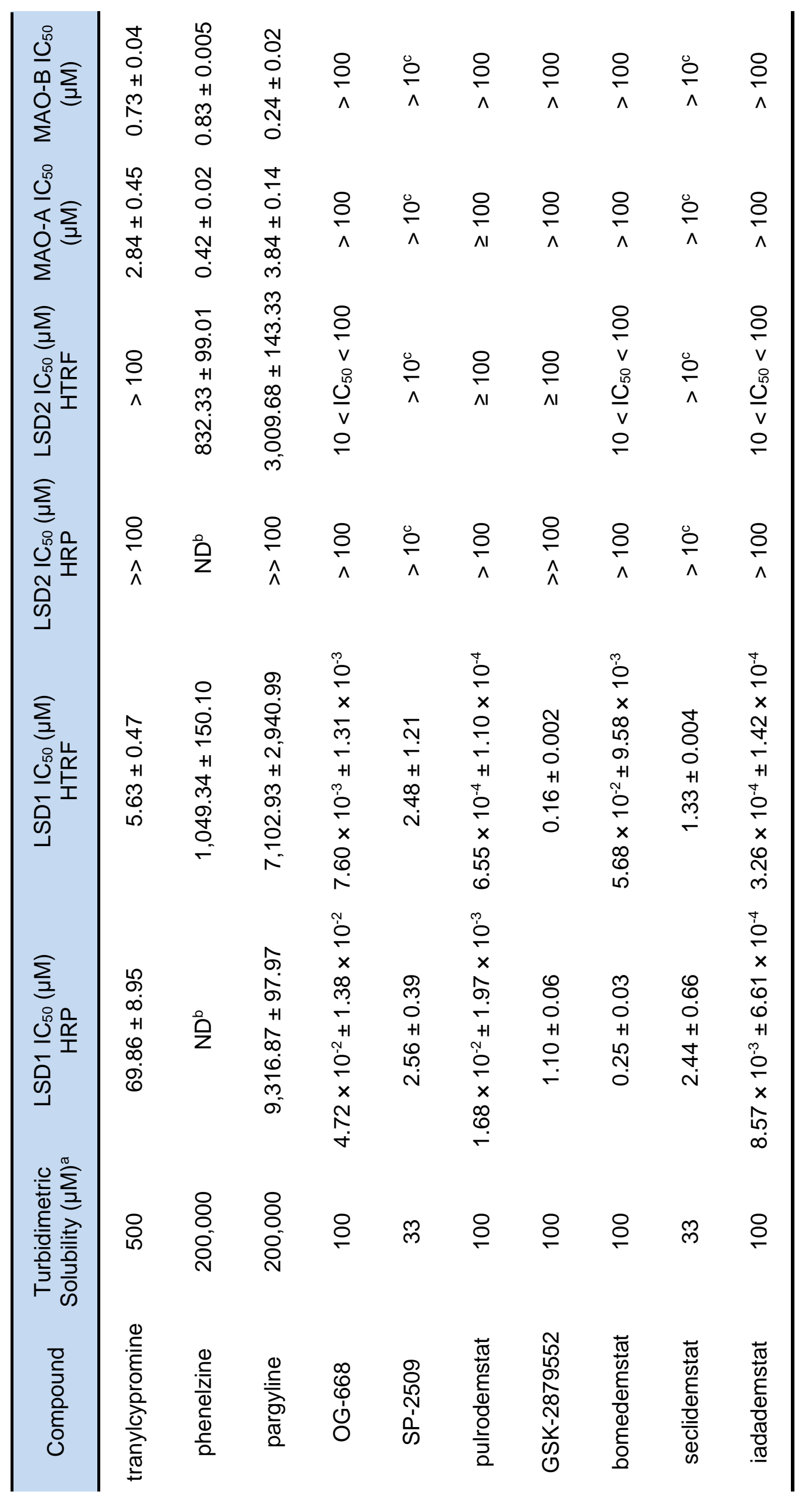




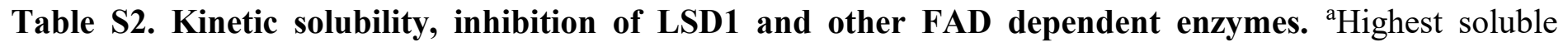
concentration. $\mathrm{ND}^{\mathrm{b}}$ not determined: the compound is fluorescent at HRP assay wavelength. ${ }^{\mathrm{c}}$ Compound not soluble at $100 \mu \mathrm{M}$. Data are represented as mean \pm St. Dev. of $\mathrm{N}=2$ or $6, \mathrm{n}=2$ or 3 . 


\begin{tabular}{cccc}
\hline Compound & LSD1 $\mathrm{K}_{\mathrm{I}}(\mu \mathrm{M})$ & LSD1 $\mathrm{k}_{\text {inact }}\left(\mathrm{sec}^{-1}\right)$ & $\mathrm{LSD} 1 \mathrm{k}_{\text {inact }} / \mathrm{K}_{\mathrm{I}}\left(\mathrm{M}^{-1} \mathrm{sec}^{-1}\right)$ \\
\hline tranylcypromine & $360.13 \pm 244.54$ & $0.011 \pm 0.004$ & $33.67 \pm 11.56$ \\
phenelzine & $\mathrm{ND}^{\mathrm{a}}$ & $\mathrm{ND}^{\mathrm{a}}$ & $\mathrm{ND}^{\mathrm{a}}$ \\
pargyline & $\mathrm{ND}^{\mathrm{b}}$ & $\mathrm{ND}^{\mathrm{b}}$ & $\mathrm{ND}^{\mathrm{b}}$ \\
OG-668 & $0.626 \pm 0.186$ & $0.016 \pm 0.005$ & $2.57 \times 10^{4} \pm 1.04 \times 10^{3}$ \\
GSK-2879552 & $2.137 \pm 1.267$ & $0.013 \pm 0.003$ & $6.73 \times 10^{3} \pm 2.43 \times 10^{3}$ \\
bomedemstat & $1.189 \pm 0.435$ & $0.018 \pm 0.001$ & $1.63 \times 10^{4} \pm 6.68 \times 10^{3}$ \\
iadademstat & $0.017 \pm 0.001$ & $0.020 \pm 0.003$ & $1.19 \times 10^{6} \pm 2.39 \times 10^{5}$ \\
\hline
\end{tabular}

Table S3. Kinetic parameters of LSD1 irreversible inhibition. $\mathrm{ND}^{\mathrm{a}}$ not determined: the compound is fluorescent at HRP assay wavelength. ND ${ }^{\mathrm{b}}$ not determined: no time-dependent inhibition was observed at the tested concentrations. Data are represented as mean \pm St. Dev. of $N=2, n=1$. 


\begin{tabular}{|c|c|c|c|c|}
\hline \multicolumn{5}{|c|}{$\mathrm{EC}_{50}(\mathrm{nM})$} \\
\hline & TF1a & MOLM-13 & $\operatorname{MV}(4 ; 11)$ & $\mathrm{NCl}-\mathrm{H} 510 \mathrm{~A}$ \\
\hline Compound & \multicolumn{4}{|c|}{ Mean \pm St. Dev. } \\
\hline tranylcypromine & $>2,000$ & $>2,000$ & $>2,000$ & $>2,000$ \\
\hline phenelzine & $>2,000$ & $>2,000$ & $>2,000$ & $>2,000$ \\
\hline pargyline & $>2,000$ & $>2,000$ & $>2,000$ & $>2,000$ \\
\hline OG-668 & $1.55 \pm 0.57$ & $0.91 \pm 0.61$ & $0.48 \pm 0.15$ & $1.17 \pm 0.84$ \\
\hline SP-2509 & $1,624.50 \pm 260.92$ & $203.35 \pm 6.72$ & $422.00 \pm 42.99$ & $56.24 \pm 1.75$ \\
\hline pulrodemstat & $7.87 \pm 3.65$ & $0.96 \pm 0.51$ & $2.90 \pm 1.98$ & $1.77 \pm 0.67$ \\
\hline GSK-2879552 & $76.43 \pm 20.71$ & $40.19 \pm 0.47$ & $39.59 \pm 3.21$ & $109.48 \pm 30.72$ \\
\hline bomedemstat & $31.06 \pm 9.24$ & $5.38 \pm 0.42$ & $4.20 \pm 0.78$ & $10.46 \pm 0.73$ \\
\hline seclidemstat & $1,261.50 \pm 96.87$ & $284.65 \pm 18.03$ & $885.65 \pm 692.05$ & $184.35 \pm 0.92$ \\
\hline iadademstat & $0.09 \pm 0.04$ & $0.21 \pm 0.17$ & $0.10 \pm 0.06$ & $0.16 \pm 0.03$ \\
\hline
\end{tabular}

Table S4. EC 50 viability values for AML and SCLC cell lines. EC 50 values were calculated using the GraphPad Prism 9 software. Data are represented as mean \pm St. Dev. of $N=2, n=3$ 


\begin{tabular}{cc}
\hline \multicolumn{2}{c}{ Relative $\mathrm{EC}_{50}(\mathrm{nM})$} \\
\hline Compound & THP-1 cells \\
\hline tranylcypromine & $>2,402.00$ \\
phenelzine & $>2,000$ \\
pargyline & 0.62 \\
SP-2509 & $>2,000$ \\
pulrodemstat & 2.34 \\
GSK-2879552 & 31.23 \\
bomedemstat & 4.15 \\
seclidemstat & $>2,000$ \\
iadademstat & 0.04 \\
\hline
\end{tabular}

Table S5. EC50 differentiation values for the AML cell line THP1. EC 50 values were calculated using the GraphPad 9 Prism software. N=1 